\title{
EXPERIENCIA DE LOS HOMBRES DURANTE EL EMBARAZO DE SU ESPOSA Y NACIMIENTO DE SU HIJ O (A)
}

\author{
Men experience during Pregnancy of his wife and the birth of his child \\ *Vilma Mercedes Miranda Baquedano **Delmy Aracely Trujillo Serrano
}

\section{RESUMEN}

Antecedentes. Escasas investigaciones se realizan sobre las experiencias del hombre durante el embarazo y nacimiento de su hijo, y son tan diversas y variadas como en las mujeres. Objetivo: Conocer las experiencias de los hombres durante el embarazo de su esposa y el nacimiento de su hijo(a). Pacientes y Métodos: Estudio cualitativo realizado de marzo a mayo del año 2014 en el área de espera de la sala de Labor y Parto del Hospital Leonardo Martínez Valenzuela (HLMV), mediante entrevistas individuales a 20 hombres que esperaban el nacimiento de su hijo(a) Resultados: Las experiencias del hombre durante el embarazo de su compañera son variadas, sin embargo cuando hay deseos de ser padre se involucra más en apoyar a su esposa, el hombre apoya más en actividades del hogar y menos al asistir al control prenatal. Al existir unión sentimental, y una relación estable, son más frecuentes los signos y síntomas psicológicos o fisiológicos; con el pasar de los meses el hombre se va absteniendo de las relaciones sexuales. Discusión Conocer el sentir y pensar del hombre referente al embarazo denota la necesidad de involucrarlo en el proceso, hacerle sentir "estamos esperando un hijo" y no como, "mi mujer está embarazada".

*Profesora de la Escuela de Ciencias de la Enfermería de la Escuela Universitaria de Ciencias de la Salud (EUCS) de la Universidad Nacional Autónoma de Honduras en el Valle de Sula (UNAH - VS). Licenciada en Enfermería. Especialista en Salud Materno Perinatal. Magister en Gestión Educativa.

** Estudiante de la Carrera de Enfermería de la EUCS-UNAHVS.

Recibido: 4 de Noviembre 2,014 Aprobado 12 de Marzo 2,015
Conclusión: Los resultados destacan que los hombres tienen numerosas experiencias, cambios psicológicos, fisiológicos y adquisición de nuevos roles, desean ser parte del embarazo por lo cual deben ser educados referente al mismo.

\section{PALABRAS CLAVE}

Embarazo, hombres, parto, signos y síntomas.

\section{ABSTRACT}

History: Few investigations are conducted on the experiences of man pregnancy and birth of his child, and are as diverse and varied as in women. Objective: To know the experiences of men during his wife's pregnancy and the birth of his child. Patients and Methods: Qualitative study conducted from march to may 2014 in the waiting area of labor and delivery room Leonardo Martinez Valenzuela (HLMV) Hospital's, through individual interviews with 20 men who awaited the birth of his child. Results: The experiences of man during the pregnancy of his partner are varied, however, when desire to be a father, It becomes more involved in supporting your wife. Man relies more on household activities and less to attend the prenatal control. As there is emotional attachment, and a stable relationship, are more frequent psychological or physiological signs and symptoms; with the passing months the man stars abstaining from sex. Discussion: know the heart and mind of man, concerning 
the pregnancy indicates the need to involve them in the process and not exclude him, make him feel "are expecting a child" rather than, "my wife is pregnant." Conclusion: The results highlight that men have many experiences, psychological and physiological changes, and take on new roles, they want to be part of pregnancy and therefore should be educated regarding the same and do not exclude it.

\section{KEYWORDS}

pregnancy, men, parturition, Signs and Symptoms

\section{INTRODUCCION}

Se sabe relativamente poco respecto a los cambios psicológicos y emocionales que ocurren en el futuro padre y cómo se realiza la transformación a la paternidad, es decir, la "transición a la parentalidad"(1) mucho se ha hablado acerca de los cambios por los que atraviesa una mujer embarazada, pero hay poca información del proceso por el que transitan sus parejas. Según publica la BBC (British Broadcasting Corporation) en un estudio de 282 hombres cuyas parejas están embarazadas, estos experimentaron síntomas del embarazo como: calambres, náuseas, dolores de espalda e incluso inflamación de estómago, experimentando el fenómeno llamado Síndrome de Couvade. ${ }^{(2)}$ Tradicionalmente los hombres han sido relegados o se han confinado a sí mismos a un segundo plano durante el embarazo. En la actualidad, la tendencia hacia una paternidad más responsable e igualitaria es cada vez mayor, hay que recordar que las diversas maneras de entender la figura paterna tienen su reflejo en las distintas culturas, ${ }^{(3)}$ sin embargo, cada vez es más frecuente que se interesen en participar activamente de este proceso.

Existen pocas investigaciones que respalden sobre los cambios, experiencias o roles del hombre en esta etapa de la vida y de cómo asume la paternidad. Para el futuro papá las cosas son diferentes a la de la futura madre, aunque él acompañe los cambios que la mujer experimenta, nunca va a sentir lo que ella siente, por lo que su futuro hijo es en realidad un ser al que podrá acercarse y conectarse, pero sólo en parte. El momento del nacimiento será de gran expectativa para el papá y es realmente el momento cuando lo pueda ver, sentir, tocar, mirar y hablarle; por lo que se transformará para él en alguien más real.

En el primer trimestre experimenta reacciones diversas y variadas como en las mujeres; atraviesan por sentimientos confusos porque su foco intelectual se concentra en la paternidad inminente y no en el estado inmediato del embarazo.

En el segundo trimestre cuando perciben los movimientos del feto les causa una sensación profunda y lo sienten real, comienzan a pensar en las implicaciones de la paternidad y entra en una etapa de estabilidad. Los hombres se hacen más consientes del crecimiento del útero de su mujer, es probable que tengan pensamientos y preocupaciones igual como le ocurre a la madre, sin embargo, también se preocupan acerca de si serán buenos padres para sus hijos y si cumplirán con las expectativas de la madre.

El tercer trimestre se describe como un periodo de crisis y requiere ajustes tanto para 
la mujer como para el hombre. El trabajo de parto marca la terminación de la etapa de gestación y es la transición real al rol de padre lo que conlleva a profundos efectos en él.(4)

Los hombres presentan diferentes vivencias frente al embarazo y la paternidad, las cuales varían de acuerdo a la cultura. Una manifestación natural que puede darse en los hombres es la presencia de signos y síntomas lo que es conocido como "Síndrome de Couvade, considerando la existencia del mismo cuando el padre presenta cinco o más signos o síntomas".(5) Aun cuando no existe una teoría que explique con certeza el origen de este síndrome, algunas investigaciones científicas se han enfocado en los cambios hormonales que se desarrollan en una mujer embarazada y los han comparado con estudios realizados en hombres. El objetivo del estudio fue conocer las experiencias de los hombres durante el embarazo de su esposa y el nacimiento de su hijo (a).

\section{PACIENTES Y METODOS}

Estudio cualitativo realizado durante el periodo de marzo a mayo del 2014, en el área de espera de la sala de Labor y Parto del HLMV en la Ciudad de San Pedro Sula, Cortes, Honduras. El modo de acceso a los participantes fue haciendo visitas durante horas por la tarde en un periodo de 7 días; obteniendo una muestra de 20 participantes. Criterios de inclusión: fueron los hombres que esperaban el nacimiento de su hijo (a) en el momento que se realizó la visita. Criterios de exclusión: los hombres que no estaban esperando un hijo (a).

Los datos se investigaron a través de 9 categorías:
1. Opinión del hombre sobre el embarazo.

2. Sensaciones que experimentó al saber que sería padre.

3. Experiencia durante el embarazo.

4. Apoyo a su esposa durante el embarazo.

5. Presencia de algún signo o síntoma fisiológico o psicológico durante el embarazo.

6. Sexualidad en el embarazo.

7. Pensamientos y sentimientos a la espera del nacimiento de su hijo (a).

8. Emociones al ver a su hijo (a).

9. Rol de padre.

De estas categorías se identificaron significados comunes e interconectados según coincidencia de respuestas.

\section{RESULTADOS}

A partir de la entrevista en donde se plantearon nueve categorías relacionadas con las experiencias de los hombres durante el embarazo y nacimiento de su hijo se encontraron semejanzas en los resultados.

\section{OPINIÓN DEL HOMBRE SOBRE EL EM- BARAZO}

Ellos concuerdan en que la mujer experimenta una serie de cambios físicos y psicológicos. Opinan que el embarazo "es una serie de cambios físicos que tiene la mujer y que es la oportunidad de ambos de ser padres, lo sienten como una etapa bonita y una bendición en la vida de cada uno de ellos".

Una de las opiniones:

"Es una gran experiencia ver cómo crece la panza y ver los movimientos del bebe, es de admirar a la mujer y más cuando le tocan los dolores". 


\section{SENSACIONES EXPERIMENTADAS CUANDO SUPO QUE SERÍA PADRE}

Experimentaron diferentes sensaciones las cuales se describen a continuación; de los que sintieron alegría opinaron "que se sentían feliz porque los llamarían papá, y sentían que tendrían algo que sería suyo, que tenían que ser más responsables y dejar los amigos".

Otros experimentaron asombro: "En mi mente nunca cruzó que un hijo cambiaría mi vida y que iba entender a mi padre y ser más responsable".

Los que tenían más hijos experimentaron preocupación: "Me sentí emocionado, pero hay preocupación cuando ya hay muchos hijos". Los que tenían una relación de noviazgo experimentaron miedo: "Algo cardíaco y que el mundo se nos venía encima porque éramos novios no estaba preparado, luego alegría y agradecimiento a Dios por un hijo".

\section{EXPERIENCIAS DURANTE EL EMBA- RAZO DE SU ESPOSA}

Los resultados sobre las experiencias en el embarazo son muy variados para los hombres y estas van desde: descubrir cosas nuevas, leer, tener paciencia, asimilar cambios en el cuerpo y separaciones por los cambios de carácter en la mujer.

\section{APOYO A SU ESPOSA DURANTE EL EMBARAZO}

En esta categoría los resultados demuestran frecuentemente el apoyo en los quehaceres del hogar. Si embargo, 20\% manifestaron apoyo en el aspecto emocional y económico. Y solo el $10 \%$ manifestaron acompañarla a su control prenatal.
Una de las opiniones

"Soy muy hogareño, lavaba la ropa, pasaba pendiente de sus antojos, en todo la complacía: hacerle comida, masajes, aseo de la casa".

\section{PRESENTÓ ALGÚN SÍNTOMA FISIO- LÓGICO O PSICOLÓGICO DURANTE EL EMBARAZO}

Todos los hombres experimentaron signos y síntomas tanto fisiológicos como psicológicos y estos van desde: subir de peso, hambre, antojos, nauseas, vómitos, sueño y rechazos.

Una de las opiniones

"Subí de peso, me dio hambre, mareos, vómitos, nauseas, dolor de muelas, sueño y muchas ganas de comer coco y zapote, pollo con tajadas, arroz con leche".

"Odiaba una compañera de la nada"; y otro: "Mi suegra me caía mal".

\section{SEXUALIDAD DURANTE EL EMBA- RAZO}

La sexualidad del hombre en el embarazo según los resultados fue variada: para algunos fue mejor porque no había preocupación de embarazo, para otros fue normal, en otros disminuyó por temor a lastimar al bebe y en algunos no hubo conforme crecía el abdomen.

"Mejor porque ya no había preocupación de un embarazo siempre hubo sexo nunca tuve miedo porque averigüé y me di cuenta que no era malo"

"Optamos otras posiciones por el abdomen, lo hacíamos con mucho cuidado porque hay preocupación desde los 4 a los 8 meses, 
luego ya no, se me quito ese deseo sexual, pensé que íbamos a lastimar al bebe".

\section{PENSAMIENTOS $Y$ SENTIMIENTOS DURANTE LA ESPERA CUANDO ESPOSA DABA A LUZ A SU HIJO (A)}

Son muy variados los pensamientos y sentimientos durante la espera del nacimiento de su hijo (a) tales como alegría, miedo, preocupación, nerviosismo y felicidad. El 50\% de ellos expresó sentir miedo a la pérdida de alguno de los dos.

"Sensaciones encontradas de perder alguno de los dos, miedo, preocupación, tensión".

"Feliz y con ansiedad por verla y saber cómo están"

\section{EMOCIONES AL VER A SU HIJO (A) POR PRIMERA VEZ}

Entre los hombres entrevistados las emociones fueron de alegría por conocer a su hijo (a) los abrazaron con ternura y cuidado.

Emociones percibidas por el hombre cuando vieron su hijo:

"Alegre de conocerla (o), abrazarla (o), y sentirla (o) por completo porque antes solo imaginaba, llore de alegría y dando gracias a Dios".

"Fue el mejor regalo y la mejor alegría de mi vida, creo que es lo mejor que me ha pasado".

\section{ROL AHORA QUE ES PADRE}

El $100 \%$ de los padres entrevistados ejercen un rol de proveedor con el fin de proporcionar una mejor calidad de vida a sus hijos.
"Quiero seguir trabajando y estudiar para darle una mejor vida, un buen ejemplo e inducirla al camino del señor".

"Darle lo mejor, ya con la llegada de mi hijo (a) establecí un negocio".

\section{DISCUSION}

En la presente década el hombre es más abierto a involucrarse en el embarazo de su esposa, dejando culturas erróneas de machismo, aunque continúan teniendo menor preparación social que la mujer.

En este estudio el hombre opino que el embarazo es algo admirable, ver crecer el abdomen de su esposa durante nueve meses. Concordando con la literatura, que refiere que ellos perciben el embarazo como una serie de cambios que experimenta la mujer. ${ }^{(4)}$ Cuando se dan cuenta del embarazo y confirman que esperan un hijo(a) los lleva de inmediato a la etapa de luna de miel. ${ }^{(6)}$

Los hombres demuestran que el principal apoyo que brindan a sus mujeres durante el embarazo son los quehaceres del hogar. Es asombroso que el hombre se involucre en tareas del hogar sin importar la cultura machista que predomina en estos países centroamericanos; lo que concuerda con la literatura que así como las mujeres están insertadas a la actividad reproductiva, los hombres se ha incorporado en las actividades domésticas, cuidado de la gestación y crianza de los hijos, actividades que antes le eran ajenas. ${ }^{(7)}$ En este estudio pocos hombres expresaron preocupaciones económicas lo que contrasta con otras investigaciones en donde la necesidad de solventar los 
gastos que un hijo supone, suele producir angustia y en algunos casos culpa de no estar siendo capaces de disfrutar por completo la noticia frente a su pareja. ${ }^{(8)}$

Los signos tanto fisiológicos como psicológicos que refirieron los hombres entrevistados en esta investigación van desde: subir de peso, hambre, antojos, nauseas, vómitos, sueño y rechazos, coinciden con el Síndrome de Couvade. El cual según la literatura existe desde la antigüedad, aun no hay una teoría científica que lo respalde pero, si hay investigaciones donde se sabe que es real, una mayoría de los hombres lo presenta. ${ }^{(2)}$

Las relaciones sexuales de los hombres en este estudio se modificaron de acuerdo al crecimiento del abdomen, optando por otras posiciones, o abstinencia después del octavo mes por disminución del deseo sexual y miedo a lastimar al bebe. El miedo a lastimar al bebe, es la principal causa para que el hombre evite las relaciones sexuales según investigaciones realizadas por Von Sydow (2009) y Toole, Coghlan, Holmes y Xeutvongsa (2005), ${ }^{(9)}$ de acuerdo a estos dos estudios tanto el hombre como la mujer evitan las relaciones sexuales por miedo.

En este estudio el hombre también experimento miedo durante la espera de nacimiento de su bebe, agravado por el hecho de no poder estar en el parto, relacionándolo a perder a su pareja o a su hijo(a). Actualmente, el hombre se encuentra excluido por el personal de los servicios salud pública de Honduras, desde el control prenatal hasta el proceso del trabajo de parto, no se le permite que acompañe a la parturienta aunque demuestren interés en hacerlo, algo que se establece en las Normas Neonatales de este país y que lo escribe de la siguiente forma "favorecerá la presencia de un acompañante si la parturienta lo desea".(10) En otros países existen beneficios para el futuro padre y se hace con el objetivo de aumentar el vínculo entre padre e hijos y fomentar el rol de la paternidad. ${ }^{(7)}$ Es necesario acercar a los hombres como algo más que la pareja de la mujer, sino también como individuos con historias distintas. ${ }^{(11)}$

La felicidad y el llanto sentido por los hombres al ver a su hijo (a) en este estudio, evidencia un sentir poco permitido al hombre en nuestra cultura machista: llorar. Permitirle al hombre expresar sus emociones y relacionarse con su hijo acariciando el vientre o hablándole, hace que al momento de nacer, él o ella, pueda reconocer su voz y así establecer una conexión. Según la literatura, es conocido que el hombre no está preparado para innumerables cambios en sus vidas, por eso en las clases de cuidados prenatales, la paternidad debería ser parte del programa. ${ }^{(12)}$

Todos los hombres encuestados en esta investigación refirieron que su rol es de proveedor, para proporcionar una mejor calidad de vida a sus hijos sin pensar en otros cuidados que pueden ofrecer, lo cual concuerda con la investigación realizada por la CEPAL (2005) en hombres centroamericanos, en donde el $50.67 \%$ expresaron el rol tradicional de proveedor y el $39.04 \%$ el rol moderno que abarca otras funciones además de proveedor como ser: prodigan afecto, se involucran en la crianza de los hijos y de desempeñan como formadores. En esta investigación de la CEPAL, a nivel de país, 
Honduras presenta el mayor porcentaje de hombres asumiendo este rol tradicional con $62.42 \%$ y Costa Rica el más bajo con $39.1 \% .^{(13)}$

El rol que desempeñe el hombre durante el embarazo es vital para el futuro de la pareja y del propio hijo(a), mientras que la mujer como menciona Ramona Mercer "adopta el rol maternal por medio de cuatro fases: la anticipatoria, la informal, la personal y la formal"(6), no hay evidencias de cómo el hombre adopta el rol paterno.

Promover la paternidad responsable y comprometida ha aumentado en los últimos años; según la investigadora alemana Monica Lukesch, el papel del hombre sufrió una trasformación: él está más presente en la vida familiar, se acerca al neonato con más facilidad y manifiesta sin miedo las capacidades de cuidado y protección. ${ }^{(14)}$
En conclusión, los resultados destacan que los hombres tienen un sin fin de experiencias y cambios; psicológicos, fisiológicos y adquieren nuevos roles, desean ser parte del embarazo en todos los sentidos por lo cual deben ser educados respecto al mismo. El conocer a fondo el sentir y pensar del hombre referente al embarazo denota la necesidad de involucrarlo en el proceso, hacerle sentir "estamos esperando un hijo" y no como, "mi mujer está embarazada".

\section{Recomendación}

A los (las) docentes, la formación de los/las estudiantes de la EUCS-UNAHVS en el conocimiento y aplicación de las Normas de Atención Materno Neonatal, y a la carrera de Derecho a proponer leyes gozar del acompañamiento de su pareja durante el nacimiento de su hijo (a) y en casos especiales de complicación materno- neonatal permiso personal.

\section{BIBLIOGRAFÍA}

1. Maldonado M .El padre en la Etapa Perinatal. Perinatal Reprodud Human. [Citado el 2014, Mayo 12] 2008; 22 (2) Pg. 146- 150. Disponible en: http://www.sumedico.com/documentos/25_documento.pdf.

2. Matrón de Murcia, J. Reflexología en la Maternidad, El Síndrome de Couvade. España. [Citado 2014 junio 15 ]. Disponible en: http://www.maternityreflexology.net/?categoryld=14253

3. Guzmán E. Los Hombres Avanzan hacia una Paternidad Igualitaria $y$
3. Responsable. El Nuevo Diario. 17.marzo.2012. Internacional [Citado 2014 Junio 2] Disponible en: http://elnuevodiario.com.do/app/article. aspx $? \mathrm{id}=278984$

4. Reeder S, Martin L, Koniak D. Enfermería Materno Infantil. $17^{\circ}$ ed. México DF: McGraw-Hill; 1995. Pg. 316-318

5. Bottino Y, Galilea P, Leiza P, Magnone L, Oroz B. Incidencia del Síndrome de Couvade. Montevideo. 2012. [citado 2014 Julio 5]. Disponible en: https://www.colibri.udelar.edu.uy/handle/123456789/2392 
6. Tommey AM. Modelo y Teorías de Enfermería. $4^{\circ}$ ed. Harcourt; 2005. Pg. 130-145

7. Hernández J, Quevedo D, Ramos A. Vivencia de los Adolescentes Varones frente al Embarazo. Bogotá. 2008 [citado 2014 Mayo 4] Disponible en: http://www.javeriana.edu.co/biblos/tesis/enfermeria/tesis13.pdf

8. Rivera R, Ceciliano Y. Cultura Masculinidad y Paternidad. 2nd ed. San José: FLACSO; 2004. Pg.8

9. Sapien JS, Córdova DI. Comportamiento Sexual de Varones Durante el Embarazo: Casos en la Ciudad de México D.F. Terapia Psicológica [Internet]. [Citado 2014, mayo 30] 2011; 29(2):185-190 Disponible en:

http://www.scielo.cl/scielo.php?pi$d=S 0718-48082011000200005 \&$ script=sci_arttext

10. Secretaria de Salud. Normas para la Atención Materno Neonatal. Tegucigalpa, Junio, 2010.Pg.52.

11. Maroto Navarro G, Castaño López E,
García Calvente M del Mar, Hidalgo Ruzzante N, Mateo Rodríguez I.

Paternidad y Servicios de Salud. Rev. Esp. Salud Pública [revista en Internet]. [Citado 2014 agosto 23] 2009; 83(2): 267-278. Disponible en: http://scielo.isciii.es/scielo.php?script=sci_arttext\&pi$d=S 1135-57272009000200010 \&$ Ing $=e s$.

12. Gabbe S, Niebyl J, Simpson J. Obstetricia. $4^{\circ}$ ed. Editorial Marban; 2007. Pg. 155.

13. CEPAL (Masculinidad y factores asociados al comportamiento de los hombres en Centro América. 2005; Pag.169. [Citado Mayo 7 del 2014 ]. Disponible en: http://www.berdingune.euskadi.eus/contenidos/informacion/material/es_gizonduz/adjuntos/Masculinidad $\% 20 \mathrm{y} \% 20$ factores $\% 20$ socioculturales $\%$ 20asociados\%20al\%20comportamiento \%20de\%20los\%20hombres\%20estudio \%20en\%20cua.PDF

14. Guana M, Cappadona R, Di Paolo AM, Pellegrini MG, Piga MD, Vicario $M$. Enfermería Ginecoobstétrica 2 ed. México D.F: Editorial McGraw - Hill; 2009. Pg. 210-215. 